\title{
Qualitative performance characteristics differentiate dementia with Lewy bodies and Alzheimer's disease
}

\author{
E K Doubleday, J S Snowden, A R Varma, D Neary
}

J Neurol Neurosurg Psychiatry 2002;72:602-607

See end of article for authors' affiliations

Correspondence to Dr J Snowden, Cerebral Function Unit, Greater Manchester Neuroscience Centre, Hope Hospital, Salford M6 8HD, UK julie.snowden@man.ac.uk

Received 9 April 2001 In final revised form 5 October 2001 Accepted

18 October 2001

\begin{abstract}
Objectives: To determine whether dementia with Lewy bodies (DLB) and Alzheimer's disease (AD) can be differentiated on the basis of qualitative performance characteristics during neuropsychological evaluation.

Methods: Forty one patients with clinically defined DLB were matched with 26 patients with AD for age, illness duration, nature and severity of cognitive deficits, and regional blood flow distribution on SPECT. The presence or absence of a set of qualitative performance characteristics, observed and recorded during the patients' initial cognitive evaluation, was identified by retrospective analysis of patients' records and the groups compared.

Results: Inattention, visual distractibility, impairments in establishing and shifting mental set, incoherence, confabulatory responses, perseveration, and intrusions were significantly more common in DLB than AD. Intrusions were particularly common in DLB, occurring in $78 \%$ of the group. They included externally cued intrusions arising from the visual environment, a feature never seen in AD. In a stepwise logistic regression analysis impaired mental set shifting, perseveration, and the presence of intrusions correctly classified $79 \%$ of patients.

Conclusion: It is possible to differentiate DLB and AD on the basis of qualitative features of performance. As many features are amenable to detection at clinical interview, they ought to contribute to clinicians' diagnostic armoury, leading to improved clinical recognition of DLB.
\end{abstract}

$\mathrm{T}$ he current diagnostic criteria for "probable" dementia with Lewy bodies (DLB) require the presence of cognitive impairment, together with two of the following: hallucinations, fluctuations in mental state, and extrapyramidal signs. ${ }^{1}$ Cognitive impairment may be the most prominent feature ${ }^{2-4}$ Comparative studies of DLB and Alzheimer's disease $(\mathrm{AD})$ have suggested that memory impairment is less severe in DLB than $\mathrm{AD}^{, 5-7}$ whereas visuoperceptual, visuospatial, and constructional abilities are more impaired.$^{5-68-13}$ Nevertheless, impairments in perception, spatial function, constructional abilities, and memory occur in both DLB and AD, so that consideration of the severity of dysfunction may have limited value in the diagnosis of an individual patient in a clinical setting. As yet, no unique neuropsychological pattern has been identified in DLB that clearly differentiates it from AD. Neuroimaging also has limited differentiating value. Although some studies have found that patients with DLB more commonly have reduced perfusion in the occipital lobes than patients with $\mathrm{AD},{ }^{14}{ }^{15}$ others have failed to find any significant differences between the patterns of regional cerebral blood flow distribution. ${ }^{16}{ }^{17}$

In view of the combination of cortical and prominent subcortical pathology in $\mathrm{DLB}^{18}$ and the characteristic fluctuating mental state, patients with DLB might be expected to share neuropsychological characteristics of other confusional states-for example, those caused by metabolic encephalopathies-in which there is both cortical and subcortical dysfunction. Patients in confusional states typically show poor sustained and selective attention, they are easily distracted from the task at hand, and they have difficulties in establishing, maintaining, and shifting mental set. ${ }^{19}{ }^{20}$ Responses are perseverative and may include intrusions from irrelevant stimuli. ${ }^{20}$ Additionally, there is marked fluctuation in the severity of deficits from moment to moment and from day to day. ${ }^{19} 20$

There is evidence that patients with DLB perform worse than patients with $\mathrm{AD}$ on formal tests of sustained, selective, and divided attention. ${ }^{621} 22$ It might be anticipated that impairments in attention and fluctuations in mental state ought to be reflected in different qualitative patterns of behaviour and test performance in DLB compared with AD. The purpose of the present study was to test the prediction that patients with DLB and those with $\mathrm{AD}$, matched for overall severity and nature of cognitive deficits, will exhibit qualitative differences in their pattern of performance and that patients with DLB will show performance characteristics akin to those of metabolic confusional states. The identification of such qualitative characteristics ought to be of value in the clinical differentiation of DLB and AD.

\section{METHODS}

Patients

Dementia with Lewy bodies group

The study group comprised 41 consecutive patients, referred to a regional diagnostic dementia clinic, who exhibited clinical features of DLB. ${ }^{1}$ Twenty six patients (63\%) presented initially with cognitive and/or behavioural changes, and thus fulfilled diagnostic criteria for DLB as strictly defined. ${ }^{1}$ The remaining 15 patients $(37 \%)$ had developed mental symptoms in the course of established and long standing parkinsonism, but were otherwise clinically similar to the 26 patients. At the time of investigation $71 \%$ of the total group had visual hallucinations and $71 \%$ were reported by relatives to fluctuate in their mental state from day to day or during the course of a day. Extrapyramidal signs were present in $93 \%$ of patients on neurological examination. Patients in whom hallucinations had coincided with an alteration in medication for parkinsonism were not included in the study. The clinical diagnosis of

Abbreviations: DLB, dementia with Lewy bodies; AD, Alzheimer's disease; SPECT, single photon emission tomography; MMSE, mini mental state examination; CDR, clinical dementia rating 
Table 1 Demographic and clinical characteristics

\begin{tabular}{lll}
\hline & DLB & AD \\
\hline Sex F:M & $15: 26$ & $14: 12$ \\
Age at onset (mean y (SD)) & $68(5)$ & $65(7)$ \\
Duration (mean y (SD)) & $2(1)$ & $3(1)$ \\
Education & & \\
$\quad<12$ years & 33 & 21 \\
$\quad>12$ years & 8 & 5 \\
MMSE/30 (mean (SD)) & $15(8)$ & $14(7)$ \\
CDR (mean (SD)) & $2(1)$ & $1(1)$ \\
\hline & &
\end{tabular}

DLB has subsequently been confirmed in four of the 41 patients at necropsy.

The presenting cognitive symptom in $46 \%$ of patients with DLB was of memory impairment and in 5\% of difficulties in language expression. The remaining 49\% presented with a combination of memory, visuoperceptual, and language disturbances. Functional imaging using single photon emission tomography (SPECT) disclosed the presence of posterior hemispheric abnormalities in $94 \%$ of patients with DLB; in $41 \%$ these were selective and in 53\% they were combined with anterior hemispheric abnormalities. Only 3\% of patients showed selective abnormalities in the anterior hemispheres. In 3\% of patients SPECT findings were reported to be normal. Demographic and clinical features are summarised in table 1 .

\section{Alzheimer's disease group}

Twenty six patients with $\mathrm{AD}$, attending the regional diagnostic dementia clinic, served as a comparison group. Patients exhibited impairments in memory and other domains of cognitive functioning, in the context of physical wellbeing and no impairment of consciousness, in keeping with current clinical criteria for $\mathrm{AD}^{23}$ The diagnosis of $\mathrm{AD}$ has subsequently been confirmed in three of the 26 patients at necropsy. In no patient with $\mathrm{AD}$ was there a history of fluctuating mental state or of visual hallucinations.

The patients with $\mathrm{AD}$ were selected to be as closely matched as possible to the DLB group on a range of demographic and clinical variables (table 1). The AD group did not differ significantly from the DLB group in age, illness duration, years of education, overall severity of cognitive impairment as measured by the mini mental state examination (MMSE), ${ }^{24}$ and degree of functional impairment, as determined by the clinical dementia rating scale (CDR). ${ }^{25}$ In $65 \%$ of patients with $\mathrm{AD}$ the presenting cognitive symptom was of memory impairment, in $8 \%$ difficulties in language expression, and in $4 \%$ visuoperceptual disturbance. The remaining $23 \%$ of patients presented with a combination of memory, visual, and language symptoms. The nature of presenting cognitive symptoms did not differ significantly in the AD group compared with the DLB group.

Fewer patients with AD (27\%) than patients with DLB exhibited extrapyramidal signs on neurological examination $\left(\chi^{2}=31.2, p=0.000\right)$. SPECT imaging showed the presence of posterior hemispheric abnormalities in $92 \%$ of patients with $\mathrm{AD}$, in $37 \%$ this being selective and in 55\% combined with anterior hemispheric abnormalities. No patient showed selective anterior hemispheric abnormalities. In $9 \%$ of patients images were reported to be normal. The distribution of SPECT abnormalities in the AD group did not differ significantly from that of the DLB group.

\section{Method of evaluation}

At the time of their initial clinical neurological assessment all patients had undergone a neuropsychological evaluation using a locally constructed cognitive screening instrument, designed to provide a profile of performance across a wide range of cognitive functions: language, perception, spatial abilities, praxis, memory, and frontal/executive functions (table 2). The instrument, which has been previously described ${ }^{26}$ bears some similarities to the CAMDEX, ${ }^{27}$ although it emphasises the qualitative analysis of patients' performance, such as error type and behaviour during testing, in addition to accuracy scores. Cognitive profiles elicited by the instrument show a strong relation to findings on functional imaging, ${ }^{16}$ and in conjunction with historical and neurological data have yielded a 97\% accuracy of clinical diagnosis in 200 pathologically verified cases of dementia in this centre.

Qualitative aspects of patients' behaviour, found during the course of the testing session, which lasts about 1 hour, are systematically recorded by trained examiners. Features are reported only as present or absent, in view of the difficulty in determining severity.

Features include:

- General inattention: inability to maintain vigilance and attention during the test period.

- Distraction: (a) visual-that is, attending and responding to irrelevant visual stimuli in the immediate environment (for example, during the test, patient comments at an inappropriate time on an object on the shelf); (b) auditory-that is, attending and responding to irrelevant auditory stimuli in the immediate environment (for example, during the test, patient comments irrelevantly on phone ringing in adjacent room).

- Impaired mental set establishment—-that is, impairment in initial engagement in test procedure.

- Impaired mental set shifting - that is, impairment in the smooth and appropriate sequential transition from one task to the next.

Table 2 Cognitive tests constituting the neuropsychological profile assessing each domain of cognitive functioning

\begin{tabular}{|c|c|c|c|}
\hline \multirow[b]{2}{*}{ Domain } & \multirow[b]{2}{*}{ Neuropsychological tasks upon which ratings based } & \multicolumn{2}{|c|}{ Median rating } \\
\hline & & DLB & $A D$ \\
\hline Memory & $\begin{array}{l}\text { Immediate and delayed free recall, cued recall and recognition of a } \\
\text { story }(/ 12) \text {, recalling and recognising famous faces after a delay }(/ 9) \text {, } \\
\text { orientation for time and place }(/ 10)\end{array}$ & 0 & 0 \\
\hline Language & $\begin{array}{l}\text { Conversational speech, series speech, naming to confrontation and from } \\
\text { description, word repetition, reading, writing, spelling }\end{array}$ & 1 & 1 \\
\hline Perception & Identification of 10 line drawings and nine famous faces & 2 & 2 \\
\hline Spatial function & $\begin{array}{l}\text { Tracing road map, locating the centre of a circle, line bisection, copying } \\
\text { drawings, geographical localisation }\end{array}$ & 1 & 1 \\
\hline Executive function & Picture sequencing, copying motor hand sequences, Weigl's blocks (/9) & 0 & 1 \\
\hline
\end{tabular}


- Incoherent line of thought-that is, verbal responses that are tangential, illogical, irrational departures from the designated subject or topic of conversation.

- Confabulation-that is, introduction of fictional elaborations in test responses (for example, in recalling a story, patient adds extraneous, elaborate elements).

- Perseveration-that is, repetition of the immediately preceding verbal or motor response (for example, patient correctly names picture as "scissors" but then also names next picture as "scissors").

- Intrusions - that is, irrelevant responses which are: (a) related to previously presented test material. These within test intrusions may occur from (1) interference of earlier memory test material in memory tasks (memory interference), for example, elements from one story included in the patient's account of a second story, (2) interference of previous tasks in non-memory verbal tasks (language interference), for example, in reciting months, the patient says "England" (patient had previously been asked to name the country), or (3) interference of previous motor actions in a later motor task (motor interference); $(b)$ semantically associated with the test material (associative intrusions), for example, in an animal category fluency task, patient says "donkey", followed by "Bethlehem"; (c) not related to preceding test material, but arise from stimuli in the surrounding environment (environmentally cued intrusions), for example, public sign "fire exit" interpolated irrelevantly into an otherwise appropriate speech utterance.

The neuropsychological screening is carried out independently of clinical history taking, neurological examination, and neuroimaging and in patients in whom a clinical diagnosis is not yet made. These initial assessments were carried out by two of us (JS and AV) before and hence blind to clinical diagnosis, with a comparable balance between DLB and AD assessments for the two examiners.

The present study involved an analysis of recorded information, obtained from this initial neuropsychological screen and documented in written reports.

\section{Procedure}

The evaluation of data was carried out by an independent rater ED who had not been involved in the original assessments and had no clinical contact with the patients. The rater documented each qualitative behavioural characteristic and error type as present or absent based on the examiner's written report and the raw test data. Inattention, visual and auditory distractibility, impaired mental set establishment, and shifting were recorded as present only if those features had been explicitly reported as characterising the patients' performance. Incoherence, perseverations, confabulations, and intrusions were recorded as present based on the examiner's explicit report, supported by an examination of test errors.

Table 3 Qualitative features of performance in patients with DLB and AD

\begin{tabular}{llll}
\hline Feature & $\begin{array}{l}\text { DLB } \\
(\%)\end{array}$ & $\begin{array}{l}\text { AD } \\
(\%)\end{array}$ & $\begin{array}{l}\text { Fisher's exact } \\
\text { test } p \text { value } \\
(1 \text { tailed })\end{array}$ \\
\hline $\begin{array}{l}\text { General inattention } \\
\text { Distraction: }\end{array}$ & 17 & 0 & $0.03^{*}$ \\
$\quad$ Visual & 20 & 0 & $0.02^{*}$ \\
$\quad$ Auditory & 2 & 0 & 0.61 \\
Impaired mental set establishment & 22 & 0 & $0.008^{* *}$ \\
Impaired mental set shifting & 32 & 8 & $0.03^{*}$ \\
Incoherent line of thought & 17 & 0 & $0.03^{*}$ \\
Confabulation & 17 & 0 & $0.03^{*}$ \\
Perseveration & 68 & 27 & $0.001^{* *}$ \\
Intrusions (total) & 78 & 27 & $0.000^{* * *}$ \\
\hline${ }^{*} \mathrm{p}<0.05 ;{ }^{* *} \mathrm{p}<0.01 ;{ }^{* *} \mathrm{p}=0.000$. & & \\
\hline
\end{tabular}

Rater ED also rated each domain of cognitive function (memory, language, perception, spatial function, and executive functions) on a three point severity scale on the basis of performance across the range of tasks incorporated in the assessment (table 2). Tasks are sufficiently easy to yield ceiling level performance in healthy controls. A rating of 2 was given when no abnormality was detected. A rating of 1 signified the presence of abnormalities of minor or moderate degree-for example, paraphasic errors and spelling errors on language tasks. A rating of 0 was applied to severe, incapacitating deficits such as a total inability to name on language tasks. Ratings did not differ between DLB and AD.

A second rater who had no clinical contact with the patients and was blind to the diagnoses rated $15 \%$ of the data set, which included an equal number of patients with DLB and with $\mathrm{AD}$, to determine the interrater reliability. The average agreement between the two raters was $90 \%$, yielding a mean (and median) $\mathrm{k}$ agreement measure of 0.8 (range 0.4-1.0).

\section{Statistical analysis}

For consistency, non-parametric Fisher's exact tests were computed to compare the frequency of nominal variables, as the expected cell frequency was less than 5 in the case of many variables. A one tailed test was adopted on the basis that group differences would be expected in one direction only: symptom frequency in DLB greater than AD. Differences between groups were considered to be statistically significant if $\mathrm{p}<0.05$. A forward stepwise logistic regression analysis was conducted to determine the predictive power of independent variables. Contingency coefficients were calculated to determine the association between nominal variables.

\section{RESULTS}

\section{Comparison of DLB and AD}

Inattention, visual distractibility, impairment in establishing and shifting mental set, incoherent line of thought, confabulation, perseveration, and intrusions were all significantly more common in the DLB than the AD group (table 3 ), and many of the features were specific to DLB. The most marked group differences were in intrusions. Intrusions representing interference from previous tasks occurred in both groups to some degree, whereas environmentally cued intrusions were present only in patients with DLB (Fisher's exact test $\mathrm{p}=0.005$, fig 1). That is, patients with DLB incorporated irrelevantly into general conversation and into their test responses, the names of extraneous objects in the immediate environment or a word or phrase from a public notice on which their glance happened

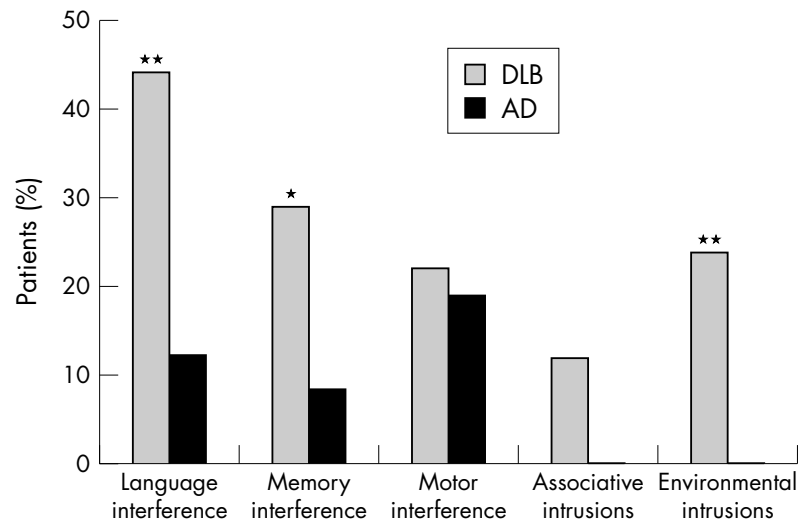

Figure 1 Percentage of patients exhibiting intrusion errors. Language, memory, and motor interference represent within test intrusions; associative intrusions are semantically related intrusions environmental intrusions are external intrusions, arising from the visual environment. * $p<0.05 ;{ }^{*}{ }^{*} p<0.01$. 
momentarily to fall (for example, question:. What programmes do you watch on television? Answer. "I watch programmes about the fire exit"). With regard to within test intrusions, both language interference (Fisher's exact test $\mathrm{p}=0.005$ ) and memory interference (Fisher's exact test $\mathrm{p}=0.03$ ) were significantly more frequent in the DLB group (fig 1), with the largest difference between DLB and AD occurring in non-memory verbal tasks, such as naming. Associative intrusions occurred only in the DLB group, although group differences did not reach formal levels of statistical significance (Fisher's exact test $\mathrm{p}=0.08$ ).

A stepwise logistic regression analysis identified impaired mental set shifting, perseveration, and the presence of intrusions as the combination of variables that best discriminated the groups. These three variables accurately classified $88 \%$ of the DLB group and $65 \%$ of the AD group, giving an overall accuracy of $79 \%$.

To determine the potential discriminating value of specific types of intrusions the generic variable of "intrusions" was removed from the analysis and a further forwards stepwise logistic regression analysis was undertaken. Language interference and perseveration classified $78 \%$ of the DLB group and $62 \%$ of the $\mathrm{AD}$ group, giving an overall classification accuracy of $72 \%$.

Feature frequencies had been determined on the basis of documented reports of individual patients by two of us (JS and AV). There was no difference in the frequency with which we documented any of the qualitative performance characteristics, indicating consistency of report across examiners.

\section{Effect of DLB presentation on performance}

Patients who develop clinical features of DLB in the context of longstanding parkinsonism do not strictly fulfil diagnostic criteria for DLB criteria as currently defined. ${ }^{1}$ When this subgroup of patients were removed from the DLB versus AD comparisons, the pattern of findings remained essentially unchanged, the only difference being that the presence of confabulation and memory interference no longer reached significance.

Direct comparisons between the subgroup of patients with DLB presenting with cognitive/behavioural change and the group presenting with parkinsonism showed no differences in the frequency of qualitative features in the two subgroups.

\section{Interrelation between qualitative features}

Given the assumption that qualitative characteristics may relate to inattention, the interrelation in patients with DLB between inattention and each of the qualitative characteristics of performance was examined using contingency coefficients. A significant association was found between the presence of general inattention and visual distraction (contingency coefficient, $p=0.000$ ), environmentally cued intrusions (contingency coefficient, $\mathrm{p}=0.03)$, and impaired mental set establishment (contingency coefficient, $\mathrm{p}=0.01$ ). No other interrelations reached significance. The association between visual distraction and environmentally cued intrusions was also examined, because both involve environmental visual stimuli. The two were not significantly related. To determine the relation between characteristics of test performance in patients with DLB and the historical report of fluctuating mental state, contingency coefficients were calculated for each variable. Fluctuating mental state was not significantly associated with the presence of any qualitative characteristics.

\section{DISCUSSION}

The study examined the hypothesis that patients with DLB would be more likely than patients with AD to exhibit qualitative performance characteristics akin to those found in metabolic confusional states. The DLB and AD groups in the present study were well matched on a range of demographic and clinical variables. They were relatively youthful, reflecting the pattern of referral to a neurological dementia clinic. They had a similar overall level of severity of cognitive impairment, a similar profile of deficits across cognitive domains and, consistent with previous reports, ${ }^{16}{ }^{17}$ a similar pattern of functional imaging abnormalities on SPECT. Nevertheless, in keeping with prediction, there were significant qualitative differences in test performance between the two groups. Patients with DLB were more likely to show overall inattention and overt distractibility from the visual environment. Despite similar levels of language impairment between groups, the DLB group had more difficulty grasping and adhering to task demands (impaired mental set establishment) and more difficulty switching from one set of test demands to another (impaired mental set shifting). They were more likely to deviate from the point in conversation (incoherence) and to produce confabulatory responses. They were also more likely to exhibit intrusion errors.

Intrusions, in the present study, significantly increased the likelihood of a patient having DLB rather than $\mathrm{AD}$ and contributed to the discrimination between the two groups. Other studies, however, have drawn attention to intrusions as a feature of $\mathrm{AD}^{28-36}$ Several factors may contribute to this apparent disparity. Firstly, currently accepted diagnostic criteria for $\mathrm{AD}$ are known to have low specificity, despite high sensitivity, ${ }^{37-39}$ so that presumed $\mathrm{AD}$ groups may in fact include patients with DLB. This is particularly plausible in early studies published before DLB achieved widespread clinical recognition. Secondly, reports of intrusions in AD are typically based on comparisons with controls, rather than other degenerative disorders. Even a few intrusions, as in the present $A D$ groups, would be likely to exceed those of the normal population. It is instructive that a study that compared language function in $\mathrm{AD}$ and acute confusional states reported fewer intrusion errors in AD than in acute confusion. ${ }^{40}$ Thirdly, studies of $\mathrm{AD}$ have typically not differentiated types of intrusion. ${ }^{28-36}$ Typically, reported intrusions in $\mathrm{AD}$ arise from earlier test material, conforming to the within test interference categories of the present study ${ }^{33}{ }^{35}{ }^{36}$ or occur in tasks specifically designed to measure inhibitory control, ${ }^{28}{ }^{29}$ such as the Stroop task ${ }^{41}$ or Hayling inhibition task. ${ }^{42}$ Intrusions of the associative type ${ }^{30}{ }^{31}{ }^{34}$ have usually arisen in list learning tasks, which are likely to be particularly vulnerable to intrusion errors. To our knowledge, environmentally cued intrusions, found in a quarter of our patients with DLB, have never been reported in $\mathrm{AD}$, which is consistent with our own finding of their absence in AD.

Environmentally cued intrusions seem to have a high specificity for DLB. Although not invariable, their presence is striking. A patient whose glance falls momentarily on a spoon lying on an adjacent table may instantly incorporate the object name into an ongoing conversation (for instance, "Yesterday we went to the spoon"). Printed words on notice boards in the patients' visual field may be similarly incorporated. In the present study, environmentally cued intrusions were reported only in the visual domain. All interviews were undertaken in a quiet test setting, so that the absence of reports of auditory intrusions might simply reflect the relative paucity of auditory compared with visual potentially distracting stimuli. Alternatively, patients with DLB might indeed be particularly susceptible to visual distraction. The issue requires prospective study.

It would be reasonable to suppose that patients who fail to maintain vigilance and attention to the testing stimuli during the assessment period, would be distractible and show intrusion errors from environmental stimuli. In support of this view, there was a strong association between reports of general inattentiveness and the presence of environmentally cued intrusions. General inattentiveness was unsurprisingly also significantly associated with reports of visual distraction. More unexpectedly though, there was no association between visual distractibility itself and the presence of intrusion errors 
from the visual environment. That is, patients whose attention was drawn to irrelevant environmental stimuli (visual distraction) did not necessarily interpolate those stimuli inappropriately into their verbal utterances.

The clinical presentation of the patients with DLB did not influence significantly qualitative performance characteristics. Patients who develop the characteristic features of DLB in the context of longstanding parkinsonism do not fulfil current diagnostic criteria for DLB as strictly defined. Nevertheless, the qualitative similarities in findings in the present study between patients presenting with cognitive change and those presenting with parkinsonism suggest that the two should be considered as a range of the same underlying disorder, as suggested by the neuropathological overlap between DLB and PD. $^{18} 43$

Some methodological issues warrant consideration. The qualitative features documented in the present study were derived from reports of neuropsychological performance at the time of patients' initial medical referral. The issue of logical circularity would arise if those same qualitative features contributed to the patients' clinical diagnosis. It is worth emphasising, however, that patients with DLB fulfil current diagnostic criteria for DLB, and patients with AD fulfil criteria for $\mathrm{AD}$ but not for DLB, independently of the performance measures documented here. Indeed the lack of correlation between relatives' historical reports of fluctuations in mental state and the measures reported here suggest that they are independent markers of the disorder.

The study was to some extent retrospective, representing an evaluation of previously acquired data. This raises the inevitable issue of reliability of data ascertainment. Nevertheless, data were acquired systematically using the same testing procedure and by the same examiners. Additionally, the data were obtained independently of the clinical history, neurological examination, and neuroimaging and before a clinical diagnosis had been made thus precluding bias, based on preconceptions about clinical diagnosis. Moreover, in view of the fact that qualitative features were defined as present only when they were explicitly documented in patients' reports, potential inaccuracies are likely to be in the direction of underestimation rather than overestimation of feature prevalence. Group differences obtained from the analyses are thus more likely to represent a conservative rather than exaggerated estimate of actual differences between DLB and AD.

The groups were closely matched for demographic variables and severity of dementia. Additional ratings of specific aspects of cognition demonstrated that the groups were broadly matched across domains as well as on the global measures. Previous studies have suggested that quantitative differences may exist between DLB and AD across cognitive domains. Memory is reported to be less impaired in DLB, whereas various studies have found visuoconstructional tasks, including clock drawing, to be more impaired, ${ }^{79^{12}}{ }^{13}$ and interestingly a different pattern of performance has been documented in the two diagnostic groups on the clock face task. ${ }^{9}$ Nevertheless, it was not the intention of this study to consider quantitative differences, and it is possible that differences similar to those previously reported ${ }^{6911}$ may have emerged on specific tests. The severity ratings serve merely to show that the qualitative differences in performance occur despite patients being broadly matched across cognitive domains.

Qualitative analysis is inevitably subjective and prone to differences in interpretation and threshold for report. Examiners in our clinic are trained to detect and report characteristics of patients' behaviour in a uniform and systematic fashion. Moreover, reports are based purely on the presence or absence of a characteristic, so are not dependent on subtle judgements of severity. The absence of difference in symptom frequency reported by different examiners suggests that the method of analysis is reliable. Nevertheless, it would be important to confirm the findings in prospective studies across different clinical centres. The patients were relatively youthful reflecting the pattern of referral to this specialist diagnostic centre. It remains to be seen whether comparable qualitative group differences occur in more elderly patient cohorts.

The absence of an association between relatives' reports of mental fluctuation and the qualitative measures reported in the study is to some extent surprising. It might have been anticipated that performance characteristics such as inattentiveness, distractibility and intrusions would cooccur with a fluctuating mental state. However, the fluctuations reported by relatives were based on observation of their relative's mental state from one day to another or during the course of the day. By contrast, the qualitative features found in our study might be anticipated to be behavioural manifestations of momentary alterations in level of arousal, over minutes or seconds. ${ }^{22}$ The association between performance characteristics and brief attentional fluctuations warrants further investigation.

In conclusion, the study confirms that patients with DLB exhibit many of the performance characteristics that are typically associated with confusional states and that these characteristics can serve to differentiate DLB from AD. The findings are especially important in the absence of a robust diagnostic imaging or biological marker for the diagnosis of DLB. They highlight features that are largely amenable to detection at clinical interview and from patient observation, providing complementary support to the clinical history and findings on neurological examination. The identification of discriminating clinical features is also increasingly important because of improved prospects for therapeutic intervention. Patients with DLB have been found to show particular benefit from cholinergic agents such as rivastigmine, ${ }^{44}$ and patients with DLB but not patients with $\mathrm{AD}$ have been shown to be susceptible to neuroleptic sensitivity syndrome..$^{45}$ The present study suggests that clinically observed inattention and distractibility, impairments in establishing and switching mental set, incoherence, confabulatory responses, perseverations, and intrusions are all strong differentiating features. Future prospective studies, using independent markers of fluctuation, such as encephalography, ought to shed more direct light on the precise relation between arousal and other aspects of attention and the elicitation of distinctive performance characteristics of confabulation, perseveration, and intrusions.

\section{ACKNOWLEDGEMENTS}

We thank Ms Zoë Gibbons for her help with interrater reliability.

\section{Authors' affiliations}

E K Doubleday, J S Snowden, A R Varma, D Neary, Greater Manchester Neuroscience Centre, Hope Hospital, Salford, UK

\section{REFERENCES}

1 McKeith IG, Galasko D, Kosaka K, et al. Consensus guidelines for the clinical and pathologic diagnosis of dementia with Lewy bodies (DLB): report of the consortium on DLB international workshop. Neurology 1996:47: 11113-24.

2 Salmon DP, Galasko D, Hansen L, et al. Neuropsychological deficits associated with diffuse Lewy body disease. Brain Cogn 1996;31:148-65

3 Förstl H, Burns A, Luthert $P$, et al. The Lewy-body variant of Alzheimer's disease: clinical and pathological findings. $\mathrm{Br} J$ Psychiatry 1993; 162:385-92.

4 Byrne EJ, Lennox G, Lowe J, et al. Diffuse Lewy body disease: clinical features in 15 cases. J Neurol Neurosurg Psychiatry 1989;52:709-17. 5 Salmon DP, Galasko D, Hansen LA, et al. Neuropsychological deficits associated with diffuse Lewy body disease. Brain Cog 1996;31:148-65.

6 Calderon J, Perry R, Erzinclioglu SW, et al. Perception, attention and working memory are disproportionately impaired in dementia with Lewy bodies and Alzheimer's disease. J Neurol Neurosurg Psychiatry 2001;70: 157-64

7 Shimomura T, Mori E, Yamashita H, et al. Cognitive loss in dementia with Lewy bodies and Alzheimer's disease. Arch Neurol 1998;55:1547-52 
8 Hansen L, Salmon D, Galasko MD, et al. The Lewy body variant of Alzheimer's disease: a clinical and pathologic entity. Neurology 1990;40:1-8.

9 Gnanalingham KK, Byrne EJ, Thornton A, et al. Motor and cognitive function in Lewy body dementia: comparison with Alzheimer's and Parkinson's diseases. J Neurol Neurosurg Psychiatry 1997;62:243-52.

10 Ala TA, Hughes LF, Kyrouac GA, et al. Pentagon copying is more impaired in dementia with Lewy bodies than in Alzheimer's disease. J Neurol Neurosurg Psychiatry 2001;70:483-8.

11 Mori E, Shimomura T, Fujimori $M$, et al. Visuoperceptual impairment in dementia with Lewy bodies. Arch Neurol 2000;57:489-93.

12 Galasko D, Katzman R, Salmon DP, et al. Clinical and neuropathological findings in Lewy body dementias. Brain Cogn 1996;31:166-75.

13 Walker Z, Allen RL, Shergill S, et al. Neuropsychological performance in Lewy body dementia and Alzheimer's disease. Br J Psychiatry 1997; 170:156-8.

14 Lobotesis K, Fenwick JD, Phipps A, et al. Occipital hypoperfusion on SPECT in dementia with Lewy bodies but not AD. Neurology 2001;56:643-9.

15 Ishii K, Yamaji S, Kitagaki H, et al. Regional cerebral blood flow difference between dementia with Lewy bodies and AD. Neurology 1999;53:413-16.

16 Neary D, Snowden JS, Shields RA, et al. Single photon emission tomography using $99 \mathrm{mTc}-\mathrm{HM}-\mathrm{PAO}$ in the investigation of dementia. $J$ Neurol Neurosurg Psychiatry 1987;50:1101-9.

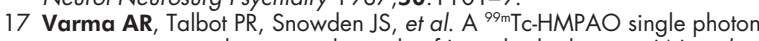
emission computed tomography study of Lewy body disease. J Neurol 1997;244:349-59.

18 Lennox G, Lowe J, Landon $M$, et al. Diffuse Lewy body disease: correlative neuropathology using anti-ubiquitin immunocytochemistry. $J$ Neurol Neurosurg Psychiatry 1989;52:1236-47.

19 Albert MS. Acute confusional states. In: Albert MS, Moss MB, eds. Geriatric neuropsychology, chapter 6. New York: The Guilford Press, 1988

20 Mendez MF. Delirium. In: Bradley WG, Daroff RB, Fenichel GM, et al, eds. Neurology in clinical practice. Vol 1. Principles of diagnosis and management, 2nd ed, chapter 4. Oxford: Butterworth-Heinemann, 1996.

21 Sahgal A, Galloway PH, McKeith IG, et al. A comparative study of attentional deficits in senile dementias of Alzheimer and Lewy body types. Dementia 1992;3:350-4.

22 Walker MP, Ayre GA, Cummings JL, et al. Quantifying fluctuation in dementia with Lewy bodies, Alzheimer's disease and vascular dementia. Neurology 2000;54:1616-24.

23 McKhann G, Drachman D, Folstein M, et al. Clinical diagnosis of Alzheimer's disease: report of the NINCDS-ADRDA work group under the auspices of Department of Health and Human Services Task Force on Alzheimer's disease. Neurology 1984;34:939-44.

24 Folstein MF, Folstein SE, McHugh PR. Mini-mental state: a practical method for grading the cognitive state of patients for the clinician. $J$ Psychiatr Res 1975;12:189-98.

25 Hughes CP, Berg L, Danziger WL, et al. A new clinical scale for the staging of dementia. Br J Psychiatry 1982;140:566-72.
26 Neary D, Snowden JS, Bowen DM, et al. Neuropsychological syndromes in presenile dementia due to cerebral atrophy. J Neurol Neurosurg Psychiatry 1986;49:163-74.

27 Roth M, Huppert FA, Tym E, et al. CAMDEX: The Cambridge examination for mental disorders of the elderly. Cambridge: Cambridge University Press, 1988.

28 Faust ME, Balota DA, Duchek JM, et al. Inhibitory control during sentence comprehension in individuals with dementia of the Alzheimer type. Brain Lang 1997;57:225-53.

29 Collette F, Van der Linden M, Salmon E. Executive dysfunction in Alzheimer's disease. Cortex 1999;35:57-72.

30 Amieva $\mathbf{H}$, Lafont S, Rainville $\mathrm{C}$, et al. Analysis of inhibitory dysfunction in patients with Alzheimer's disease and normal elderly adults in two verbal tasks. Brain Cogn 1998;37:58-60.

31 Kern RS, Van Gorp WG, Cummings JL. Confabulation in Alzheimer's disease. Brain Cogn 1992;19:172-82.

32 Spieler DH, Balota DA, Faust ME. Stroop performance in healthy younger and older adults and individuals with dementia of the Alzheimer's type. J Exp Psychol Hum Percept Perform 1994;22:461-79.

33 Marson DC, Annis SM, Mclnturff B, et al. Error behaviours associated with loss of competency in Alzheimer's disease. Neurology 1999:53:1983-92

34 Dalla Barba G, Wong C. Encoding specificity and intrusion in Alzheimer's disease and amnesia. Brain Cogn 1995;27:1-16.

35 Shindler AG, Caplan LR, Hier DB. Intrusions and perseverations. Brain Lang 1984;23:148-58.

36 Fuld PA, Katzman R, Davies P, et al. Intrusions as a sign of Alzheimer's disease: chemical and pathological verification. Ann Neurol 1982;11:155-9.

37 Lopez OL, Litvan I, Catt KE, et al. Accuracy of four clinical diagnostic criteria for the diagnosis of neurodegenerative dementias. Neurology 1999;53:1292-9

38 Varma AR, Snowden JS, Lloyd JJ, et al. Evaluation of the NINCDS-ADRDA criteria in the differentiation of Alzheimer's disease and frontotemporal dementia. J Neurol Neurosurg Psychiatry 1999;66:184-8.

39 McKeith IG, Fairburn AF, Perry RF, et al. The clinical diagnosis and misdiagnosis of senile dementia of Lewy body type (SDLT). Br J Psychiatry 1994; 165:324-32.

40 Wallesch C-W, Hundsalz A. Language function in delirium: a comparison of single word processing in acute confusional states and probable Alzheimer's disease. Brain Lang 1994;46:592-606.

41 Trenerry MR, Crosson B, DeBoe J, et al. Stroop neuropsychological screening test. USA: Psychological Assessment Resources, 1989.

42 Burgess PW, Shallice T. Response suppression, initiation and strategy use following frontal lobe lesions. Neuropsychologia 1996;34:263-73.

43 Hurtig HI, Trojanowski JQ, Galvin J, et al. Alpha-synuclein cortical Lewy bodies correlate with dementia in Parkinson's disease. Neurology 2000;54:1916-21.

44 McKeith I, Del Ser T, Spano P, et al. Efficacy of rivastigmine in dementia with Lewy bodies: a randomised, double-blind, placebo-controlled international study. Lancet 2000;356:2031-6.

45 McKeith IG, Fairburn AF, Perry RH, et al. Neuroleptic sensitivity in patients with senile dementia of Lewy body type. BN 1992;305:673-8. 